- Case Report

\title{
Epileptic Seizure Due to Disulfiram Treatment
}

\author{
Habib Erensoy*, Ahmet Emre Sargin \\ Department of Psychology, NPIstanbul Clinic, Üsküdar University, Istanbul, Turkey
}

Disulfiram has been used for the treatment of alcohol dependence for nearly 65 years and is approved by the Food and Drug Administration. It causes negative reinforcement by accumulating toxic acetaldehyde due to irreversible inhibition of aldehyde dehydrogenase. Disulfiram has very few side effects when taken without alcohol. Epileptic seizure induction is a rare side effect in therapeutic doses, and its mechanism is unknown. We present a patient with a single epileptic seizure which was thought to be due to disulfiram used in the treatment of alcohol dependence. We did not find it ethical to administer disulfiram again because the patient discontinued alcohol use and was afraid of epileptic seizures.

Keywords: Epileptic Seizures; Disulfiram; Alcohol Dependence; Alcoholism Treatment 


\section{INTRODUCTION}

Disulfiram causes accumulation of toxic acetaldehyde by inhibiting aldehyde dehydrogenase during alcohol metabolism. ${ }^{1)}$ Intoxication symptoms include flushing, nausea, vomiting, tachycardia, and hypotension. ${ }^{1)}$ If not taken with alcohol, the side effects of disulfiram include insomnia, headache, skin eruptions, and impotence. A few cases of epileptic seizures of unknown mechanism related to dosage have been reported. ${ }^{2)}$ Although rare, clinicians should be aware of the potential occurrence of seizures due to the risk of death. Patients with hypersensitivity, serious liver, kidney or respiratory failure, diabetes, and cardiovascular disease, or persons who consumed alcohol within 24 hours are strictly advised against use of disulfiram. ${ }^{1,3)}$

We present a patient with no epilepsy history who had a single seizure after taking low-dose disulfiram without alcohol.

\section{CASE REPORT}

A 44-year-old, married, financial advisor consulted the psychiatry outpatient clinic with a 4-year history of anhedonia, depressed mood, headache, and anger management problems and reported 'sometimes' drinking alcohol. He had no other disease or medication history.

He reported increasing consumption of alcohol and sometimes drank at work. He quarreled with his wife, who he blamed for being oversensitive to his alcohol consumption. On psychiatric evaluation he was alert, cooperative with the interviewer, and oriented. His mood was depressed and his affect was congruent with his mood. He had anhedonia, headaches, insomnia, decreased attention and concentration, and diminished libido. He had lost $3 \mathrm{~kg}$ due to decreased appetite. He denied suicidal ideation. Global functionality showed mild impairment (Global Assessment of Functioning score, 55; Hamilton Depression Scale [HAM-D] score, 35).

Brain magnetic resonance imaging (MRI), the electroencephalogram (EEG), and thyroid tests were normal. Venlafaxine $75 \mathrm{mg} / \mathrm{d}$ was started, and the dosage was increased to $225 \mathrm{mg} / \mathrm{d}$. He remained in partial remission for 1 year (HAM-D score, 24) but still had difficulty controlling the amount of alcohol intake. Acamprosate $666 \mathrm{mg} 3$ times daily was started in September 2013. One year later, psychomotor retardation and depressed mood due to marital problems related to alcohol consumption were noted. Fluoxetine $20 \mathrm{mg} / \mathrm{d}$ and sulpiride 50 $\mathrm{mg} / \mathrm{d}$ were started instead of venlafaxine. He again achieved partial remission in 1 month. His alcohol consumption decreased. The patient stated that he did not want to give up alcohol but wanted to decrease consumption instead. Therefore, acamprosate was restarted, but he was unable to control alcohol consumption during the following 3 months. Treatment protocol was explained to the patient and informed consent was obtained from his wife prior to treatment and disulfiram $250 \mathrm{mg} / \mathrm{d}$ was started along with fluoxetine $20 \mathrm{mg} / \mathrm{d}$. Sulpiride was stopped. One month after starting disulfiram, the patient was found unconscious at home. The EEG suggested an epileptic seizure. Occasional delta slow wave paroxysms, with 9-10 cycles per second alpha rhythm and paroxysmal activity prolonged by hyperventilation were observed (Figure 1).

Brain MRI showed no abnormality, and fluoxetine and disulfiram blood levels were $27.69 \mathrm{ng} / \mathrm{mL}$ and $5 \mathrm{ng} / \mathrm{mL}$, respectively, both of which were within the normal range (120-500 ng/mL and 50-400 ng/ $\mathrm{mL}$, respectively). No alcohol metabolite was detected in the urine analysis. All blood tests were within normal range. The epileptic seizure was thought to be related to disulfiram use and the drug was stopped. No antiepileptic medication was started by the neurologist. The patient has only been on fluoxetine $20 \mathrm{mg} / \mathrm{d}$ for the last 6 months. He has not used alcohol for the last 10 months and his mood is euthymic. An EEG performed 4 months after the epileptic seizure was reportedly normal. During this time, no antiepileptic drug was prescribed.

\section{DISCUSSION}

Seizure induction may occur on therapeutic doses of disulfiram. Glover et al. ${ }^{4)}$ stated that seizures due to disulfiram are very serious but rare and are related to the dose. In our case, the blood level of disulfiram was low, strengthening the hypothesis of epileptic seizure independent of drug dose. The patient had no history of epilepsy, a normal EEG before the seizure, an epileptic EEG just after the seizure, and finally a

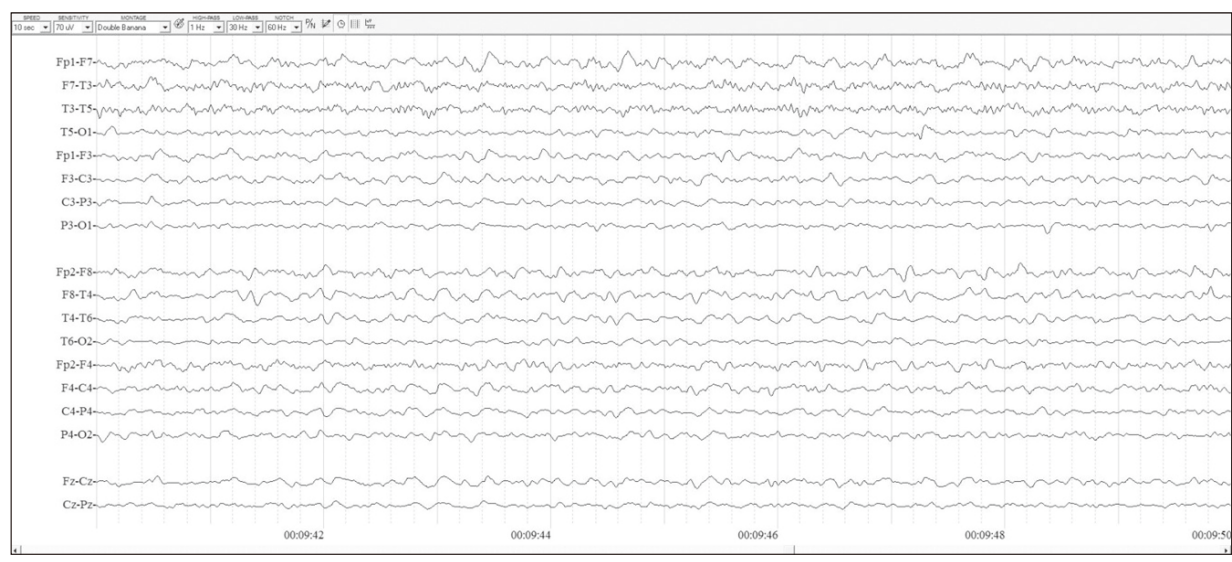

Figure 1. Electroencephalogram after loss of consciousness. 
normal EEG after drug discontinuation. These all strengthen the probability that the seizure was a reversible side effect of disulfiram. ${ }^{5)}$

Vrishabhendraiah et al. ${ }^{6}$ reported a single episode of generalized tonic-clonic seizures during treatment with disulfiram in a 35-year-old male with no past or family history of seizures. They observed generalized spike waves on the EEG during photic stimulation and no seizure recurrence for 1 month after discontinuation of disulfiram.

Kulkarni and Bairy ${ }^{7)}$ reported a prospective case series of eight subjects with alcohol dependence and various types of disulfiram-induced generalized seizures that were controlled with antiepileptics or discontinuation of disulfiram use. We did not find it ethical to give our patient disulfiram again because he discontinued alcohol use and was afraid of epileptic seizures.

\section{CONFLICT OF INTEREST}

No potential conflict of interest relevant to this article was reported.

\section{ORCID}

Habib Erensoy: https://orcid.org/0000-0002-4278-2739
Ahmet Emre Sargin: https://orcid.org/0000-0003-4634-387X

\section{REFERENCES}

1. Isik E, Uzbay T. Guncel temel ve klinik psikofarmakoloji. Ankara: Golden Medya; 2009.

2. Ugurlu TT, Sengul CB, Sengul C. Psychopharmacology of addiction. Curr Approach Psychiatry 2012;4:37-50.

3. Bankole J, Ruiz P, Galanter M. Handbook of clinical alcoholism treatment. Baltimore (MD): Lippincott Williams and Wilkins; 2003.

4. Grover S, Bhateja G, Basu D. Pharmacoprophylaxis of alcohol dependence: review and update: part I: pharmacology. Indian J Psychiatry 2007;49:19-25.

5. Price TR, Silberfarb PM. Disulfiram-induced convulsions without challenge by alcohol. J Stud Alcohol 1976;37:980-2.

6. Vrishabhendraiah SS, Gopal Das CM, Jagadeesh MK, Mruthyunjaya N. Disulfiram-induced seizures with convulsions in a young male patient: a case study. Indian J Psychiatry 2015;57:309-10.

7. Kulkarni RR, Bairy BK. Disulfiram-induced de novo convulsions without alcohol challenge: case series and review of literature. Indian J Psychol Med 2015;37:345-8. 